\title{
Remédiation cognitive des patients schizophrènes dans les pays francophones
}

\author{
M.-N. Levaux, ${ }^{1,2}$ F. Larøi, ${ }^{1}$ J.-M. Danion, ${ }^{2}$ M. Van der Linden ${ }^{1,3}$ \\ ${ }^{1}$ Unité de psychopathologie cognitive, Université de Liège, Belgique. \\ ${ }^{2}$ Service de psychiatrie I, Unité 666 Inserm, Hôpitaux universitaires de Strasbourg, 1 place de l'hôpital, BP 426, 67091 Strasbourg, France. \\ ${ }^{3}$ Unité de psychopathologie et neuropsychologie cognitive, Université de Genève, Suisse.
}

\begin{abstract}
La schizophrénie est associée à des déficits cognitifs variés qui affectent les capacités fonctionnelles des patients : relations sociales, insertion professionnelle, activités de la vie quotidienne. Les troubles cognitifs interviennent également dans le développement de certains types de symptômes cliniques (délires, hallucinations). Pour faire face à ces déficits, une prise en charge de type cognitif a vu le jour et différents programmes ont été développés. Cette revue de la littérature décrit les programmes de remédiation cognitive utilisés dans les pays francophones. Des résultats prometteurs en termes d'amélioration des fonctionnements cognitif et psychosocial ont été obtenus. Cependant, pour la plupart de ces programmes, des études de validation et d'évaluation de l'efficacité à long terme sont encore à réaliser. Plusieurs recommandations sont énoncées pour les études futures, en particulier dans le but d'améliorer la généralisation des effets de la remédiation cognitive au fonctionnement dans la vie quotidienne.
\end{abstract}

Mots clés : Schizophrénie ; Remédiation cognitive ; Déficits cognitifs ; Fonctionnement psychosocial ; Activités de la vie quotidienne

\section{Introduction}

Ces trente dernières années, une littérature abondante s'est penchée sur les déficits cognitifs (problèmes de mémoire, de langage, d'attention, de planification, de prise de décision, etc.) que l'on peut observer chez les personnes atteintes d'une schizophrénie (pour une revue récente de la littérature, cf. [1]). Il est actuellement bien établi que ces perturbations cognitives affectent les capacités fonctionnelles des patients schizophrènes : relations sociales, insertion professionnelle, activités de la vie quotidienne [2-6] Elles semblent également intervenir dans le développement de certains types de symptômes cliniques tels que la désorganisation de la pensée, les idées délirantes et les hallucinations [7,8] De plus, sur le long terme, des changements de performances cognitives sont associés à un changement correspondant dans le statut fonctionnel des patients [9]. Enfin, il apparaît que les traitements pharmacologiques ne permettent pas une diminution substantielle de ces difficultés cognitives [10, $11]$.

Ces constats ont amené les cliniciens à développer une prise en charge de type cognitif pour les personnes présentant une schizophrénie. Trois approches peuvent être distinguées [12] La première a pour objectif d'entraîner la performance sur des tâches cognitives spécifiquement affectées dans la schizophrénie (par exemple, le Wisconsin Card Sorting test) [13] La deuxième approche dite « orientée vers les processus 》 vise à restaurer ou à réorganiser les processus cognitifs déficitaires. Dans une stratégie de restauration, un ou plusieurs processus déficitaires sont entraînés via la réalisation de tâches soigneusement hiérarchisées. Dans une stratégie de réorganisation, les personnes apprennent des stratégies alternatives fondées sur des capacités préservées. La troisième approche consiste en l'implémentation d'aides externes (agendas, systèmes d'alarme, etc.) et/ou en l'aménagement de l'environnement du patient dans le but de compenser ses déficits cognitifs. Une méta-analyse récente [14] a montré que la remédiation cognitive avait un effet bénéfique (taille de l'effet : 0,41) sur divers déficits cognitifs chez les patients schizophrènes. De plus, les stratégies de réorganisation seraient plus efficaces que les stratégies de restauration [14] En particulier, elles amèneraient à un meilleur transfert des acquis vers des tâches cognitives non travaillées dans le programme de remédiation [15] Cependant, ces interventions cognitives n'ont pas conduit à une généralisation importante aux situations de la vie quotidienne des patients schizophrènes [14-18]. Or, le but d'une prise en charge cognitive est d'améliorer la qualité de vie de la personne et de réduire les handicaps consécutifs aux déficits cognitifs [19] Pour atteindre ces objectifs, la prise en charge doit nécessairement prendre en compte l'hétérogénéité des troubles cognitifs, ainsi que des difficultés dans la vie quotidienne manifestées par les personnes atteintes d'une schizophrénie.

Nous présenterons les différents programmes de remédiation cognitive qui ont été appliqués et/ou mis au point dans les pays francophones (pour un article sur ce thème, voir également [20]). Ces programmes diffèrent sur plusieurs points : le type d'approche (entraînement cognitif, apprentissage de stratégies et/ou aides 
compensatoires), le type de matériel (format papier, vidéo, interface informatisée et/ou jeux de rôles), la visée thérapeutique (cognitive, psychosociale et/ou symptomatique) et le type d'application (en groupe ou individuelle, assistée par ordinateur). En outre, ces programmes se situent sur un continuum d'individualisation de la prise en charge, allant des interventions "prêtes à porter », appliquées de la même façon à tous les patients aux études « taillées sur mesure » en fonction des difficultés spécifiques de chaque patient.

\section{Programmes de remédiation cognitive dans les pays francophones}

\section{Application en groupe}

D'un point de vue clinique, les démarches de remédiation en groupe présentent l'avantage de pouvoir travailler certaines habilités en "situation réelle " (en particulier les habilités sociales). Elles contribuent aussi à rompre l'isolement en permettant le partage des expériences personnelles et en favorisant l'entraide. Ces programmes ont généralement été élaborés afin d'être administrés à un grand nombre de personnes et ce, notamment, à des fins économiques.

\section{Integrated Psychological Treatment}

Le programme Integrated Psychological Treatment (IPT), développé en allemand [21], combine deux approches d'intervention : cognitive et psychosociale. Six sous-programmes hiérarchisés, de complexité croissante, sont proposés de manière autonome ou de manière articulée. Ils concernent les fonctions cognitives de base (différenciation cognitive), la perception sociale, la communication verbale, la gestion des émotions et les compétences sociales (habilités sociales et résolution de problèmes). Les exercices sont sous format papier, vidéo et jeux de rôles. Le programme est destiné à des groupes de 8 à 12 participants et se déroule sur une période de 9 à 12 mois, à raison d'une à trois séances par semaine d'une durée d'environ 45 à 90 minutes chacune. Une métaanalyse récente [22] portant sur 30 études réalisées dans différents pays du monde a mis en évidence son efficacité (taille des effets : 0,40 à la fin du programme et 0,52 après une période de suivi d'environ 8 mois), ainsi que l'utilité de combiner les deux approches (cognitive et psychosociale). De la sorte, le fonctionnement cognitif et social, ainsi que les symptômes psychiatriques des patients schizophrènes s'amélioraient après un programme IPT comparé à des soins standards. Cependant, Velligan et al. [23] ont souligné le manque de spécificité des effets bénéfiques de l'IPT sur la cognition.

Une version française [24] a été validée au Québec suite à une implementation multisites [25] En outre, une analyse de satisfaction de la part des participants et des intervenants a suscité des modifications et des ajouts ciblés pour permettre la généralisation et le maintien des effets dans la vie quotidienne (exercices à domicile, sorties d'intégration et relances). Il est à noter que pour rencontrer ces objectifs, il semble essentiel de constituer des groupes présentant des déficits cognitifs et des difficultés dans la vie quotidienne les plus homogènes possibles. En effet, le programme peut être adapté en fonction des caractéristiques d'un groupe et non de chaque participant pris individuellement.

\section{Remédiation de la mémoire autobiographique}

La mémoire autobiographique (ou mémoire des souvenirs d'événements personnellement vécus), dans ses liens avec la capacité de se projeter dans le futur, est intimement liée à la construction de l'identité personnelle (ou « self »), ainsi qu'à la planification et à la réalisation des buts [26, 27]. De nombreuses études montrent que la remémoration consciente d'événements personnels est perturbée chez les patients schizophrènes [28-30].

Dans ce contexte, une équipe belge [31] a développé une thérapie fondée sur l'entraînement à la remémoration consciente de souvenirs personnellement vécus. Une étude préliminaire a été réalisée sur un groupe de 15 patients schizophrènes.

Durant 10 semaines, les patients devaient journellement rapporter dans un carnet un événement personnel spécifique, ainsi que les pensées et les émotions qui y étaient associées. Lors de chaque session (d'une durée de 90 minutes), ils s'interrogeaient par deux sur le contenu du carnet de l'autre qu'ils avaient échangé préalablement. Des exercices de stimulation de pensées sur l'identité personnelle étaient également proposés aux patients. Suite à l'application de la thérapie et trois mois après, les patients rapportaient plus d'événements spécifiques (mesurés par un test de mémoire autobiographique). Cependant, l'intervention n'a pas conduit à des effets bénéfiques sur les symptômes dépressifs et anxieux et sur d'autres fonctions cognitives. Ces résultats préliminaires sont néanmoins encourageants et suggèrent de poursuivre la mise au point et l'évaluation spécifique de ce type de programme.

\section{Application assistée par ordinateur}

Les thérapies assistées par ordinateur présentent l'avantage de proposer un entraînement cognitif standardisé et hiérarchisé fournissant un feedback précis et immédiat au participant. De plus, la personne est confrontée à une évaluation qu'elle peut ressentir comme étant plus neutre que celle du thérapeute. 


\section{REHACOM}

Le programme REHACOM (REHAbilitation COMputarisée) est une interface informatisée développée en allemand et initialement conçue pour la remédiation cognitive de patients souffrant de lésions cérébrales. Dans une étude préliminaire de validation entreprise par une équipe française [32] le programme a été appliqué de manière individuelle à 30 patients schizophrènes au cours de sept séances successives, à intervalle d'une semaine. Il consiste en quatre modules indépendants visant des fonctions spécifiques : l'attention/concentration, la mémoire topographique, le raisonnement logique et les fonctions exécutives (via un exercice de shopping virtuel). Les modalités d'utilisation sont simples (quelques touches et une mannette) et attrayantes (interface ludique composée d'objets animés et inanimés). Les exercices sont construits en différents niveaux hiérarchisés, adaptables en fonction des performances du participant, lequel reçoit un feedback précis et immédiat. Les séances durent environ 20 minutes ( 25 pour le module « shopping »).

Les résultats de l'étude se sont montrés concluants pour les fonctions cognitives entraînées, le fonctionnement psychosocial (mesures d'habilité de résolution de problèmes et d'autonomie sociale) et la symptomatologie psychopathologique. Néanmoins, une étude de validation complète (notamment en y incluant un groupe de contrôle) s'avère nécessaire.

\section{Application mixte : en groupe et individuelle}

\section{Programme de renforcement de l'autonomie et des capacités sociales}

Le Programme de renforcement de l'autonomie et des capacités sociales (PRACS) a été mis au point par une équipe française [33] pour faire face aux limites présentées par les programmes existants de remédiation des compétences sociales : non-prise en compte de la vie quotidienne des personnes et absence de prise en charge individualisée. Ce programme est fondé sur deux types d'approche : la psychoéducation et la remédiation cognitive. Son objectif est de trouver des solutions aux problèmes concrets de la vie quotidienne des patients et de les aider à réaliser des projets personnalisés. Quatre domaines de compétences sociales peuvent être travaillés (programme fermé ou à la carte) : gérer son argent, gérer son temps, développer ses capacités de communication et ses loisirs, améliorer sa présentation. Le programme doit permettre aux patients de prendre conscience de possibles améliorations dans ces domaines de compétence et les amener à un niveau d'autonomie compatible avec une vie sociale et relationnelle satisfaisante. Il présente l'intérêt de combiner des séances en groupe (quatre ou huit participants) et des séances individuelles. Les premières ont pour but de permettre aux patients d'acquérir des savoir-faire par différentes techniques d'apprentissage dont certaines sont inspirées de la remédiation cognitive (utilisation d'un agenda, résolution de problèmes, jeux de rôle, prescription de tâches à domicile, etc.). Durant ces séances de groupe, des informations générales concernant les différents domaines de compétence travaillés sont également dispensées. Les séances individuelles permettent au patient de définir et de travailler un ou plusieurs objectifs personnels à partir des apprentissages reçus en groupe. Le programme dure trois à quatre mois (environ 30 séances au total) à raison de deux séances par semaine (une individuelle de 30 minutes et une en groupe d'une durée de deux heures). Des séances individuelles de soutien sont ajoutées lorsque les séances individuelles ne suffisent pas pour atteindre les objectifs et permettre une mise en place des apprentissages dans la vie quotidienne. Des séances de rappel en groupe sont également prévues à raison d'une rencontre tous les six mois pendant deux ans.

Une étude de validation [34] a été réalisée chez 18 patients schizophrènes appariés à un groupe contrôle. Des améliorations suite au PRACS ont été montrées sur le niveau d'autonomie sociale, la qualité de vie, la symptomatologie (négative et non spécifique) et sur certains tests cognitifs évaluant les fonctions attentionnelles, mnésique et exécutives des patients. Un point intéressant à soulever est que l'aspect très concret des objectifs définis à la hauteur des possibilités et des attentes des participants en séances individuelles favorise des modifications rapides dans le quotidien des personnes (gain visible et évolutif). Le programme renforce également l'estime de soi et le sentiment de responsabilité du patient. Un suivi a été réalisé sur sept des 18 patients schizophrènes pour mesurer le maintien des acquis évalués deux ans après le programme. Une stabilité des acquis a été observée pour les aspects cognitifs, psychosociaux et cliniques.

\section{Application individuelle}

D'un point de vue clinique, les démarches individualisées présentent l'avantage de permettre une évaluation détaillée et une prise en charge personnalisée des déficits cognitifs et des problèmes rencontrés par la personne dans sa vie quotidienne.

\section{Remédiation d'un déficit de la théorie de l'esprit}

Un déficit de la «théorie de l'esprit », c'est-à-dire la capacité à comprendre et à attribuer des états mentaux à autrui (intentions, émotions et désirs) a fréquemment été observé dans la schizophrénie. Ce déficit contribue vraisemblablement à divers symptômes, tels que les idées délirantes ou les difficultés d'interactions sociales. 
Une équipe française [35] a réalisé une étude préliminaire visant à améliorer la « théorie de l'esprit » chez huit patients schizophrènes. Le matériel utilisé pour la remédiation était composé des scènes vidéo montrant deux ou trois personnes en interaction et dont l'état mental devait être identifié et analysé par le participant. Deux sessions, chacune comprenant le visionnage de six films et d'une durée d'une heure, étaient administrées à une semaine d'intervalle. Comparés à un groupe de contrôle, les patients montraient, après la thérapie, une amélioration des performances dans une tâche d'attribution d'états mentaux à autrui et des scores à un questionnaire sur la capacité de communication. En revanche, la symptomatologie générale ne montrait pas d'amélioration. Les auteurs relèvent en outre le manque d'éléments permettant de déterminer les mécanismes spécifiques ou généraux impliqués dans l'amélioration des performances des participants.

Remédiation du processus de « source monitoring "

Les processus appelés «contrôle de la source » (« source monitoring ») permettent de discriminer l'origine interne ou externe d'une information (imaginée ou perçue). Ils constituent l'un des facteurs impliqués dans les hallucinations $[3,36]$.

Sur base de ce constat, une équipe suisse [37] a adopté une approche de réorganisation des processus de «source monitoring " chez un patient schizophrène souffrant d'intrusions de pensées attribuées à tort à une personne extérieure. Le type d'erreur commis par le patient évalué en préremédiation (attribuer ses propres mots au thérapeute) constituait la cible de la thérapie : une stratégie d'association entre les mots autogénérés et des souvenirs personnels significatifs durant la phase d'encodage a été apprise au patient durant 11 semaines (6 heures de traitement au total). Des exercices à domicile étaient également demandés au patient pour permettre une généralisation de la stratégie à la vie quotidienne. Le patient a amélioré ses performances relatives à l'objectif-cible de la remédiation (reconnaissance de mots autogénérés en lien avec le nombre de souvenirs personnels rappelés, avec une évaluation selon un paradigme en lignes de base multiples) et les progrès se sont maintenus un mois après la fin de la thérapie. De plus, une amélioration sur une échelle mesurant les hallucinations auditives du patient a également été observée et elle s'est maintenue un an après. Cette étude pilote encourage à l'utilisation de la remédiation de certains processus cognitifs dans le traitement des hallucinations.

\section{Programme RECOS}

Le programme RECOS (REmédiation Cognitive pour patients présentant une Schizophrénie ou un trouble associé) a été développé en français par une équipe suisse [38]. Le programme a la particularité de prendre en compte les déficits cognitifs en lien avec la symptomatologie clinique, ainsi que les répercussions fonctionnelles de ces déficits chez chaque patient schizophrène considéré individuellement. Le programme se déroule en différentes étapes. Il commence par une phase élaborée d'évaluation : cognitive (tests cognitifs standardisés), clinique (symptomatologie, estime de soi, plaintes cognitives subjectives, troubles du cours de la pensée, adaptation sociale) et fonctionnelle (analyses des difficultés quotidiennes). Ensuite, une séance de psychoéducation est réalisée durant laquelle des objectifs individualisés sont définis. Vient ensuite le programme de remédiation cognitive en tant que tel, qui, selon les difficultés cognitives rencontrées par le patient, peut viser le raisonnement, la mémoire verbale, la mémoire et l'attention visuospatiales, la mémoire de travail et l'attention sélective. Un sixième module portant sur la mémoire de la source est en cours de développement. Les exercices sont d'abord de type papier-crayon permettant au patient et au thérapeute de développer des stratégies de résolution de problèmes. Celles-ci sont ensuite mises en application par le patient durant des exercices informatisés. La motivation du patient est sollicitée par le niveau croissant de difficulté des exercices (renforcement positif), par des feedbacks automatisés et des informations psychoéducatives. Des critères de performance sont établis pour passer d'un niveau au suivant. Enfin, une réévaluation cognitive et clinique similaire à celle réalisée en prétraitement est réintroduite après l'intervention. À ce jour, il n'existe pas d'étude de validation de ce programme.

\section{Approche de remédiation cognitive individualisée et centrée sur les activités de la vie quotidienne}

Une approche qui cible de manière encore plus spécifique des objectifs de remédiation cognitive en lien avec les difficultés dans la vie quotidienne présentées par chaque patient pris individuellement a été développée conjointement par une équipe belge et une équipe française. Prenant en compte l'importante hétérogénéité des déficits cognitifs et des difficultés dans la vie quotidienne, cette approche se fonde essentiellement sur une méthodologie en cas uniques. Les auteurs préconisent tout d'abord une définition d'objectifs ciblés et concrets, en relation directe avec le fonctionnement de la personne dans sa vie quotidienne. Sur cette base, il s'agit ensuite d'élaborer des stratégies de remédiation cognitive visant spécifiquement à rencontrer ces objectifs. Pour cela, une phase d'évaluation prétraitement a pour but, d'une part, d'identifier la ou les composantes cognitives déficitaires, d'éventuelles capacités préservées et de possibles facteurs d'optimisation et, d'autre part, d'interpréter les difficultés dans la vie quotidienne à partir du profil de déficits cognitif du patient. Suite à cette évaluation et aux deux types d'informations recueillies (profil cognitif et analyse des difficultés quotidiennes du patient), des stratégies de remédiation sont construites pour répondre aux différents objectifs fonctionnels établis en 
concertation avec le patient. Pour évaluer l'efficacité et la spécificité des interventions, des lignes de base, adaptées à une approche en cas unique, sont conçues selon un paradigme en lignes de base multiples et comportent à la fois des mesures cognitives, des mesures du fonctionnement quotidien et des mesures de la symptomatologie psychopathologique. À ce jour, plusieurs études de cas ont été réalisées selon cette approche.

Dans une première étude publiée [39], un patient schizophrène présentait un déficit spécifique de mise à jour en mémoire de travail (à savoir une difficulté à modifier de façon flexible et continue le contenu de la mémoire de travail en fonction de l'arrivée de nouvelles informations). Ce déficit a été mis en relation avec les difficultés rencontrées par le patient dans sa vie quotidienne : suivre une conversation ou une émission de radio, comprendre le déroulement d'un récit lu. Sur la base du profil cognitif et des plaintes du patient, un programme de remédiation de la fonction de mise à jour de la mémoire de travail [40] a été appliqué. Dans un premier temps, le but était de rendre le patient capable d'acquérir des stratégies cognitives soulageant la charge mentale subie par la mémoire de travail et ce, via des exercices de mises à jour de complexité croissante. Dans un deuxième temps, le patient a appris à appliquer ces stratégies dans des tâches de la vie quotidienne impliquant la fonction de mise à jour. Les résultats de l'étude ont montré l'efficacité et la spécificité de l'intervention centrée sur la mise à jour et la généralisation des effets bénéfiques aux activités de la vie quotidienne du patient.

Une deuxième étude $[41,42]$ a été réalisée chez deux patientes schizophrènes présentant différentes difficultés dans la vie quotidienne, lesquelles ont nécessité la mise en place d'interventions spécifiques. Face à des difficultés de mémorisation de textes (livres, articles) communes aux deux patientes, une stratégie d'organisation du matériel à l'encodage et à la récupération leur a été apprise. Une intervention centrée sur la mémoire de travail a été proposée afin de diminuer les difficultés exprimées par les deux patientes pour suivre les conversations, ainsi que les émissions TV ou radios. Pour l'une des patientes qui se plaignait d'oublier des rendez-vous ou des tâches à effectuer dans sa vie quotidienne, l'utilisation d'un carnet-mémoire (agenda) lui a été apprise. Pour l'autre patiente qui éprouvait des difficultés à apprendre les informations factuelles nécessaires à l'obtention du permis de conduire théorique, une technique d'apprentissage sans erreur, censée exploiter les capacités préservées de mémoire implicite, a été appliquée. Les effets bénéfiques de ce type de technique d'apprentissage ont été démontrés dans plusieurs études chez des patients schizophrènes [43-46] Enfin, cette patiente présentait également des symptômes de vérification compulsive (vérification de la porte d'entrée, du gaz et des lumières). Afin de réduire ces compulsions, une technique mise au point par Tallis [47] et visant à rendre le comportement de vérification plus distinctif a été administrée. Ces différentes interventions centrées sur les plaintes ont été parfaitement acceptées par les patientes. Suite à la thérapie, elles étaient autonomes dans l'application des stratégies apprises, montraient des améliorations sur toutes les lignes de base et exprimaient des progrès dans leur vie quotidienne pour tous les objectifs ciblés. La spécificité des interventions a été démontrée par le fait que d'autres fonctions cognitives déficitaires, mais non prises en charge (fonctions attentionnelles) ne montraient pas d'amélioration chez les deux patientes. Les interventions futures selon une telle approche devraient se fonder sur des évaluations plus détaillées afin d'explorer plus finement les processus cognitifs impliqués dans les activités de la vie quotidienne.

\section{Aides cognitives}

Un autre type d'intervention cognitive consiste à structurer l'environnement du patient et/ou à lui fournir des aides externes dans le but de compenser les difficultés qu'il rencontre dans sa vie quotidienne. Contrairement à ce qu'on observe chez les patients victimes d'une lésion cérébrale acquise (en neuropsychologie), peu d'études consacrées aux patients schizophrènes se sont penchées sur l'utilisation de supports externes (listes d'activités à vérifier, alarmes, messages textes, etc.) et de stratégies compensatoires d'aménagement de l'environnement (supprimer les stimuli non pertinents pour la tâche, mémos placés à des endroits stratégiques, etc.). Les résultats de quelques travaux suggèrent cependant qu'il est possible, via ce type d'approche, d'améliorer le fonctionnement quotidien des patients, ainsi que leur qualité de vie [48, 49] Avec les progrès de la technologie, des assistants cognitifs numériques, spécifiquement adaptés aux capacités d'apprentissage des patients, devraient pouvoir être utilisés pour aider la personne dans certaines de ses activités quotidiennes : se rappeler la prise des médicaments, planifier ses activités, organiser et respecter ses rendez-vous, etc. (pour une revue de la littérature, cf. [50]).

Une équipe francoquébécoise travaille actuellement sur la mise au point d'un agenda électronique pour assister les patients souffrant de schizophrénie dans leur vie quotidienne et ce, en collaboration avec leurs aidants (ergothérapeutes ou travailleurs sociaux). Une première étude [51] a été réalisée afin de vérifier la facilité d'utilisation de l'outil développé et son impact sur le fonctionnement quotidien de quatre patients schizophrènes. L'agenda permet au patient de consulter sa liste d'activités à réaliser dans les quatre heures suivantes, d'évaluer la fréquence et l'intensité de ses symptômes, d'évaluer l'outil lui-même et de demander une assistance. Les activités $\mathrm{du}$ patient sont programmées à partir d'un autre agenda utilisé par l'aidant qui suit le patient dans sa vie quotidienne. Les résultats de cette étude montrent que l'outil est fonctionnel, mais que certaines améliorations doivent être apportées pour augmenter son utilisation chez le patient et l'aidant. Des bénéfices tirés de l'utilisation de cette aide externe ont été perçus par les aidants (moyen de communication entre les deux partenaires et suivi 
écologique de l'état de fonctionnement de la personne) et les patients eux-mêmes (aspect sécurisant, confiance en soi).

\section{Recommandations pour le futur}

Les différents programmes de remédiation cognitive utilisés dans les pays francophones ont conduit à des résultats prometteurs en termes d'amélioration $\mathrm{du}$ fonctionnement cognitif et psychosocial des personnes atteintes d'une schizophrénie. Cependant, beaucoup de ces programmes sont encore dans une phase préliminaire et nécessitent un travail de validation et d'évaluation du maintien des bénéfices à long terme.

Les méthodes utilisées dans ces programmes d'intervention sont très variées et il s'avérerait utile d'analyser de façon systématique les indications spécifiques de chaque programme ou sous-programme en fonction de différents objectifs d'intervention. De même, il serait également important d'examiner dans quelle mesure ces différents programmes ou sous-programmes peuvent s'articuler pour permettre une prise en charge la plus complète, mais aussi la plus parcimonieuse possible.

Quoi qu'il en soit, le but ultime de toute remédiation cognitive est de permettre au patient schizophrène d'atteindre une certaine autonomie dans sa vie quotidienne, sociale et/ou professionnelle. Dans cette perspective, il s'agit de comprendre la nature des difficultés manifestées par les patients dans les situations quotidiennes. Une approche particulièrement bien adaptée à ce type d'objectif consiste à évaluer le patient dans des situations proches des activités de la vie quotidienne (les simulations d'activités) ou encore à l'observer directement dans la réalisation d'activités réelles. Il importe néanmoins que cette évaluation écologique et les analyses de tâches qui en découlent s'effectuent en maintenant un cadre interprétatif cognitif. Autrement dit, l'évaluation des troubles dans la vie quotidienne suppose l'énoncé d'un ensemble d'hypothèses quant aux processus impliqués et, en fonction des résultats obtenus aux examens cognitifs, l'émission d'hypothèses quant à ceux qui se trouvent perturbés [3]. En d'autres termes, un simple repérage phénoménologique du déficit ou une analyse fonctionnelle des relations existant entre les événements antécédents, la conduite émise et les événements conséquents ne suffisent pas pour comprendre de quel désordre sous-jacent dépend le trouble. Une identification plus précise des processus cognitifs impliqués dans des activités spécifiques de la vie quotidienne devrait conduire à une meilleure sélection des stratégies à adopter en fonction de différents types de difficultés quotidiennes et des différents types de déficits cognitifs. Une compréhension imparfaite de la nature des difficultés quotidiennes rencontrées par les patients schizophrènes pourrait d'ailleurs expliquer pourquoi la taille des effets d'une remédiation cognitive n'a pas augmenté au fil des années [14].

\section{Références}

[1] Fioravanti M, Carlone O, Vitale B, Cinti ME, Clare L. A meta-analysis of cognitive deficits in adults with a diagnosis of schizophrenia. Neuropsychol Rev 2005;15:73-95.

[2] Green MF. What are the functional consequences of neurocognitive deficits in schizophrenia? Am J Psychiatry 1996;153:321-30.

[3] Larøi F, Van der Linden M, Levaux MN. In: Une approche cognitive de la schizophrénie. In: Van der Linden M, Ceschi G editors. Traité de psychopathologie cognitive, (tome 2). Marseille: Solal; 2008. p. 225-48.

[4] McGurk SR, Meltzer HY. The role of cognition in vocational functioning in schizophrenia. Schizophr Res 2000;45:175-84.

[5] Rempfer MV, Hamera EK, Brown CE, Cromwell RL. The relations between cognition and the independent living skills of shopping in people with schizophrenia. Psychiatry Res 2003;117:103-12.

[6] Semkovska M, Bédart M-A, Godbout L, Limoge F, Stip E. Assessment of executive dysfunction during activities of daily living in schizophrenia. Schizophr Res 2004;69:289-300.

[7] Baxter RD, Liddle PF. Neuropsychological deficits associated with schizophrenic syndromes. Schizophr Res 1998;30:239-49.

[8] Liddle PF, Morris DL. Schizophrenic syndromes and frontal lobe performance. Br J Psychiatry 1991;158:340-5.

[9] Matza LS, Buchanan R, Purdon S, Brewster-Jordan J, Zhao Y, Revicki DA. Measuring changes in functional status among patients with schizophrenia: The link with cognitive impairment. Schizophr Bull 2006;32:666-78.

[10] Keefe RS, Silva SG, Perkins DO, Lieberman JA. The effects of atypical antipsychotic drugs on neurocognitive impairment in schizophrenia: A review and meta-analysis. Schizophr Bull 1999;25:201-22.

[11] Stip E. Cognition, schizophrénie et effets des antipsychotiques : le point de vue d'un laboratoire de recherche clinique. Encéphale 2006;32: 341-50.

[12] Van der Linden M, Seron X, Coyette F. In: La prise en charge des troubles exécutifs. In: Seron X, Van der Linden M editors. Traité de neuropsychologie clinique (tome 2). Marseille: Solal; 2000. p. 253-68.

[13] Bellack AS, Blanchard JJ, Murphy P, Podell K. Generalization effects of training on the Wisconsin Card Sorting test for schizophrenia patients. Schizophr Res 1996;19:189-94.

[14] McGurk SR, Twamley EW, Sitzer DI, McHugo GJ, Mueser KT. A metaanalysis of cognitive remediation in schizophrenia. Am $J$ Psychiatry 2007; 164:1791-802. 
Published in: EMC - Psychiatrie (2009), 37-295-E-12.

Status: Postprint (Author's version)

[15] Krabbendam L, Aleman A. Cognitive rehabilitation in schizophrenia: A quantitative analysis of controlled studies. Psychopharmacology (Berl) 2003;169:376-82.

[16] MedaliaA, Revheim N, Casey M. Remediation of memory disorders in schizophrenia. Psychol Med 2000;30:1451-9.

[17] Suslow T, Schonauer K, Arolt V. Attention training in the cognitive rehabilitation of schizophrenic patients: A review of efficacy studies. Acta Psychiatr Scand 2001;103:15-23.

[18] Pilling S, Bebbington P, Kuipers E, Garety P, Geddes J, Martindale B, et al. Psychological treatment in schizophrenia: II. Meta-analyses of randomized controlled trials of social skills training and cognitive remediation. Psychol Med 2002;32:783-91.

[19] World Health Organization. International classification of impairments, disabilities and handicaps. Geneva: WHO; 1980.

[20] Demily C, Franck N. Remédiation cognitive dans la schizophrénie. EMC (Elsevier Masson SAS, Paris), Psychiatrie, 37-820-A-55, 2008 $: 4 \mathrm{p}$

[21] Brenner HD, Hodel B, Roder V, Corrigan P. Treatment of cognitive dysfunctions and behavioral deficits in schizophrenia. Schizophr Bull 1992;18:21-4.

[22] Roder V, Mueller DR, Mueser KT, Brenner HD. Integrated psychological therapy (IPT) for schizophrenia: Is it effective? Schizophr Bull 2006;32(suppll):S81-S93.

[23] Velligan DI, Kern RS, Gold JM. Cognitive rehabilitation in schizophrenia and the putative role of motivation and expectancies. Schizophr Bull 2006;32:474-85

[24] Pomini V, Neis L, Brenner HD, Hodel B, Roder V. Thérapie psychologique des schizophrénies. Sprimont: Mardaga éditeurs; 1998.

[25] Briand C, Bélanger R, Hamel V, Stip E, Reinhartz D, Lalonde P, et al. Implantation multisite du programme Integrated Psychological Treatment (IPT) pour les personnes souffrant de schizophrénie. Élaboration d'une version renouvelée. Santé Ment Que 2005;30:73-95.

[26] Conway MA. Memory and the self. J Mem Lang 2005;52:594-628.

[27] Tulving E. Memory and consciousness. Can Psychol 1985;26:1-2.

[28] Danion JM, Rizzo L, Bruant A. Functional mechanisms underlying impaired recognition memory and conscious awareness in patients with schizophrenia. Arch Gen Psychiatry 1999;56:639-44.

[29] d'Argembeau A, Raffard S, Van der Linden M. Remembering the past and imagining the future in schizophrenia. $J$ Abnorm Psychol 2008; 117:247-51.

[30] Huron C, Danion J-M. Impairment of constructive memory in schizophrenia. Int Clin Psychopharmacol 2002;17:127-33.

[31] Blairy S, Neumann A, Nutthals F, Pierret L, Collet D, Philippot P. Improvement of autobiographical memory in schizophrenia patients following a cognitive intervention: A preliminary study. Psychopathology 2008;41:388-96

[32] Cochet A, Saoud M, Gabriele S, Broallier V, El Asmar C, Daléry J, et al. Impact de la remédiation cognitive dans la schizophrénie sur les stratégies de résolution de problèmes et l'autonomie sociale: Utilisation du logiciel rehacom. Encéphale 2006;32:189-95.

[33] Hervieux C, Gendron A-M, Lançon C, Martano B, Umidon G. A new psycho-educational programme, Pracs (programme for reinforcing autonomy and social skill). Inf Psychiatr 2007;83:277-83

[34] Hervieux C. Programme de renforcement de l'autonomie et des capacités sociales, [thèse de doctorat en psychologie non publiée], Université Aix-Marseille 1, Université de Provence, Marseille, 2007.

[35] Kayser N, Sarfati Y, Besche C, Hardy-Baylé M-C. Elaboration of a rehabilitation method based on a pathogenetic hypothesis of "theory of mind" impairment in schizophrenia. Neuropsychol Rehabil 2006;16: 83-95.

[36] Bentall RP. The illusion of reality: A review and integration of psychological research on hallucinations. Psychol Bull 1999;107: 82-95.

[37] Favrod J, Vianin P, Pomini V, Mast FW. A first step toward cognitive remediation of voices : A case study. Cogn Behav Ther 2006 ;35:159-63

[38] Vianin P, Bircher R, Deppen P, Jaugey L, Lamouille A-S, Sarrasin-Bruchez P. Programme de remédiation cognitive pour patients présentant une schizophrénie ou un trouble associé. Manuel du thérapeute. Charleroi: Socrate éditions Promarex; 2007.

[39] Levaux MN, Vezzaro J, Lar0i F, Offerlin-Meyer I, Danion J-M, Van der Linden M. Cognitive rehabilitation of the updating subcomponent of working memory in schizophrenia: A case study. Neuropsychol Rehabil 2008Jun5:1. [Epub ahead of print].

[40] Duval J, Coyette F, Seron X. Rehabilitation of the central executive component of working memory: a reorganisation approach to a single case. Neuropsychol Rehabil 2008;18:430-60.

[41] Larøi F, Van der Linden M, Fonteneau B. An everyday life approach to cognitive remediation in schizophrenia: A single case study. $13^{\text {th }}$ Winter Workshop on Schizophrenia Research, Davos, 4-10 février 2006.

[42] Levaux M-N, Fonteneau B, Lar0i F, Offerlin-Meyer I, Danion J-M, Van der Linden M. An everyday life approach to cognitive rehabilitation in two schizophrenia patients. Soumis; 2008.

[43] O'Carroll RE, Russell HH, Lawrie SM, Johnstone EC. Errorless learning and the cognitive rehabilitation of memory-impaired schizophrenic patients. Psychol Med 1999;29:105-12.

[44] Kern RS, Liberman RP, Kopelwicz A, Mintz J, Green MF. Application of "errorless learning" for improving work performance in persons with schizophrenia. Am J Psychiatry 2002;159:1921-6.

[45] Kern RS, Green MF, Mintz J, Liberman RR Does "errorless learning" compensate for neurocognitive impairments in the work 
Published in: EMC - Psychiatrie (2009), 37-295-E-12.

Status: Postprint (Author's version)

rehabilitation of persons with schizophrenia? Psychol Med 2003;33:433-42.

[46] Kern RS, Green MF, Mitchell S, Kopelwicz A, Mintz J, Liberman RP. Extensions of errorless learning for social problem-solving deficits in schizophrenia. Am J Psychiatry 2005;162:513-9.

[47] Tallis F. Doubt reduction using distinctive stimuli as a treatment for compulsive checking: An exploratory investigation. Clin Psychol Psychother 1993;1:45-52.

[48] Velligan DI, Prihoda TJ, Ritch JL, Maples N, Bow-Thomas CC, Dassori A. A randomized single-blind pilot study of compensatory strategies in schizophrenia outpatients. Schizophr Bull 2002;28: 283-92.

[49] Pijnenborg GH, Withaar FK, Evans JJ, Van den Bosch RJ, Brouwer WH. SMS text messages as a prosthetic aid in the cognitive rehabilitation of schizophrenia. Rehabil Psychol 2007;52: 236-40.

[50] Sablier J, Stip E, Frank N. Cognitive remediation and cognitive assistive technologies in schizophrenia. Encéphale 2008 (10.1016/j.encep.2008.02.010)

[51] Sablier J, Stip E, Franck N, Giroux S, Pigot H, Moreau JF, et al. Étude de convivialité de l'utilisation d'un agenda électronique par des personnes souffrant de schizophrénie. Santé Ment Que 2007;32:209-24. 